\title{
Social Work in Teen Addiction Correction Services Research Under the New Situation
}

\author{
Shiyong Zheng ${ }^{1, a}$,Weili Guan, b, ${ }^{2,}$, Biqing Li ${ }^{3, \text { a }}$, QIN De-ze ${ }^{1, a}$ \\ ${ }^{1}$ School of Computer Science and Information Engineering, Hezhou university, Hezhou \\ Guangxi 542899, China; \\ ${ }^{2}$ College of Information Engineering, Nanning university, Nanning Guangxi 530200, China; \\ ${ }^{3}$ School of Mechanical and Electronic Engineering, Hezhou university, Hezhou Guangxi \\ 542899, China \\ ajanliful@163.com, ${ }^{\text {b }}$ 13508326@qq.com \\ Corresponding Author: Weili Guan
}

Keywords: Teenager, Internet, Internet addiction, Social work, Service mode

\begin{abstract}
With the rapid development of science and technology, and better living standard of people, Internet having features of low cost, large information source and rapid speed, plays an important role in common people's life. Internet brings a lot of convenience while at the same time it causes series of social problems. Under the new situation, teen Internet addiction index is higher and higher. To solve Internet addiction problem needs every side to intervene.
\end{abstract}

\section{Introduction}

According to the survey under the new situation, it can be found that many unhealthy Internet information resources easily to distort teenagers' Three Views, namely world view, life outlook and values. At the same time, addiction into Internet games or other affiliated products for a long time will make the young form worse values such as violence and money worships, which makes them have no interest in learning at all and they prefer to use violent way to solve problems. What is worse, they probably do illegal things. To this point, social work's intervention into teen Internet addiction is more and more important and much more difficult.

\section{Teen Internet addiction problems analysis}

\subsection{Teenagers}

Adolescence, between childhood and adulthood, is a period during the process of human development. During this period, people will experience a period of puberty, which is the sexual maturation process. People also gradually form stable personality, and their unique world outlook and methodology are gradually shaped. Teenagers are those who are in adolescence. Their will and judgment are weaker than those of the adult because they are immature. When facing with new things, they prone to generate curiosity. However, they cannot completely control the bad mood and bad action caused by curiosity. When the teenagers are induced by unhealthy information, they easily get lost and choose a wrong way. China Youth Association for Network investigation shows that Internet addiction is serious among junior high school students, accounting for $38.3 \%$ of young Internet users, the vast majority of online game addiction. At present, Internet addicted teenagers are mainly to play games, whose activities and purpose are simple.

\subsection{Internet}

Internet is the network into a vast network series, among these networks there being a common protocol, connected to each other, between the agreement to form a single, huge international network logically. The emergence of the Internet is a revolution in human communications technology. Internet is the basis of the information society. 
As of December 2014, China's Internet users accounted for about half of China's total population, and the Internet penetration rate has been raised to $47.9 \%$. With the rapid development of the Internet,younger and younger children access to the Internet. Young Internet users account for high proportion, equivalent to one fifth of the country's Internet users. The cost of network is low and it is open to all, so that it is really hard to supervise. Many regulatory loopholes exist on the network, so that some criminals take advantage of and do bad things such as cyber threats phishing, hacker attacks, phishing sites, viruses, Trojans, and other malicious programs. There are a lot of violence, pornography and other unhealthy content on online media.

\subsection{Internet addiction}

Internet addiction means that a person is addicted into network for a long time, having both strong mental and action dependence on it. According to "Internet Addiction Report" released by the China Youth Internet Association three criteria are (1) that Internet users feel happier in the virtual world than in real life or have more self-realization; (2) Internet access time is always longer than that expected. Once the network is cut off, negative emotions occur; (3) they conceal their online behavior or Internet time to their guardians or teachers. To comply with any one of the three conditions, it is possible that the Internet users have Internet addiction to certain extent, it is determined that it belongs to the "Internet addiction"

\section{Under the New Situation of Social Work in Teen Addiction Correction Services}

\subsection{Family therapy}

Under the new situation, social work's service interaction into internet addicted teenagers, family therapy is extremely important. Therefore, establishing a good relationship between family structure has a significant impact on adolescent addiction treatment work. Family therapy was first proposed by the famous American psychotherapist Virginia.Saya. the 1960s American scholar S.Minuchin and his colleagues developed the family therapy to a mature extent, becoming structural family therapy mode. Structural family therapy mode mainly emphasizes the relationship between the internet addicted teenagers and the family structure. Various studies show that adolescent Internet addiction is closely related to family education. Those parents who are busy in earning money will spend little time in conducting their children to form correct personalities and grow up in a right way, thus, these children are usually eccentric and paranoid and easier to be addicted into internet. As a result, it is necessary to establish a scientific rearing style. Besides, a good filiation should be built up because in the traditional family structure, father is often a symbol of authority, while the father does not play a good role in growth process of the Internet-addicted children at all, which becomes the most great effect.

\subsection{Social support}

Among the service moles, social support affects widely. There is doubt that solving the Internet addiction problem really needs social support. The society can give material or spirit support to Internet-addicted teenagers to help them overcome their problems in accordance with their needs. It is found that in the 1960s social support was applied in the process of Rose V. Spect family therapy. It is proved that social support is good for teenagers to form the ability to deal with problems and ability to judge. Social work of curing teenager Internet addiction requires to strengthen education in citizens' social ethics and their own moral to improve their self-cultivation and ideological consciousness. At the same time. All social members should participate in and make a contribution on establishing a good environment for teenagers to grow up. Administrative organs should strengthen the management of social networking agencies, eliminate illegal network organizations, and strictly limit minors online behavior. Meanwhile, they should strengthen efforts to combat cyber crimes, regulate the Internet security order, and clean network environment. Family and school work together to correctly guild teenagers to use the Internet. Step by step, teach teenagers to identify and stay away from pornographic, violent and criminal network information. There is no doubt that administrative organization must strengthen the management of Internet addiction treatment institution, perfect theory system of Internet addiction treatment and get rid of Internet addiction in a scientific way. Conduct abundant recreational activities to distract teenagers' 
attention from Internet. And it can also increase teenagers' comprehensive quality and ability. The country should focus on the innovative production of network cultural products to make positive and sound network cultural products be predominant and cultivate a group of state holding network cultural enterprises.

\subsection{Behavior therapy mode}

Behavior therapy mode has obvious effect in intervening into teenager Internet addiction. From the perspective of cognition, teenagers should improve their self-cultivation and ideological awareness, judging things in a subjective and objective angle. Just as the proverb says: the young are strong, the country is strong; the young are weak, the country is weak. Teenagers should be based on the new situation, set up correct values, make use of Internet in a scientific way, improve technology innovative ability and try to be a promising person.

\section{Conclusion}

Internet is a double-edged knife for vast young. It brings us more advantages or more disadvantages? This is up to how we use it. Network, as a new tool for information exchange serves for people. However, it offers great chances and challenge as well. As long as teachers, parents and all society members know how to occupy the network position, guide the teenagers but not strongly forbid them and be initiative but not passive, then it is possible that teenagers can create new knowledge by high imagination in virtual network space and improve their cognition ability and ideological awareness, forming scientific network habits, by which network immunity of teenagers can be enhanced.

This work is supported the following fund :

Guangxi science and technology development projects, Tourism security key technology and development of new products research (No 1355009-9);

Project of scientific research and technology development project of Hezhou: "Design and implementation of agricultural products e-commerce platform based on Semantic Technology" (No,Hekeneng 1506006);

Guangxi science and technology development plan (No 11107006-13 \&\&No 12118017-10A);

Nanning scientific research and technology development project(No 20121033);

Nanning science and technology development plan project of Yongning district(No 20130332D);

Scientific research project of hezhou university: "The intelligent home system Implementation Research based on the Internet of Things technology" (No 2014YBZK10);

"Research and development of E-government platform based on mobile terminal" (No 2015ZZZK03);

Reform in Education project of hezhou university: "Internet of things"(No hzxytszy201501);

"Research on application personnel training model of Communications for SMEs"(No hzxyjg201525);

Master degree discipline construction scientific research and Cultivation Project: "Research on the social work service of the teenagers' Network Addiction"(No 2015SHGZ005) \&\&"Research on the intelligent development of rural tourism in Guangxi based on the Internet of things" (No 2015MTA16);

Nanling corridor of ethnic culture research base of Open Fund Project: "Nanling National corridor costumes digital display design" (No 2015kf27);

\section{References}

[1] CNNIC 35th "China Internet Development Statistics Report". China Internet Network Information Center, 2015.02

[2] Chinese NPC network: The Eleventh National People's Congress Committee Lecture the 15th Lecture on China's Internet Development and Management. 
[3] China Youth Association for Network: "Youth Internet Addiction Report".2010.02

[4] Wen Jun:"Social Work mode: Theory and Applications" Higher Education Press.2010.03

[5] Xu Su: Human Behavior and Social Environment. Social Sciences Academic Press, 2003.06 